\title{
The split feasibility problems in an infinite dimensional space
}

\author{
Mingliang Zhang \\ School of Mathematics and Statistics, Henan University, Kaifeng 475000, China.
}

Communicated by X. Qin

\begin{abstract}
The purpose of this article is to investigate the approximation of common solutions of fixed point and split feasibility problems. A viscosity iterative algorithm is introduced and studied for this approximation problem. Strong convergence theorems are established in an infinite dimensional real Hilbert space. (C)2017 All rights reserved.
\end{abstract}

Keywords: Image reconstruction, approximation solution, viscosity method, split feasibility problem. 2010 MSC: 47J20, 65J15.

\section{Introduction}

Let $A$ be an $M$ by $N$ matrix. Let $C \in R^{N}$ and $Q \in R^{M}$ be nonempty closed convex sets. Let Proj ${ }_{C}{ }^{N}$ and $\operatorname{Proj}_{Q}^{R^{M}}$ be the orthogonal projections onto $C$ and $Q$, respectively. Recall that the split feasibility problem by Censor and Elfving [7] is to find $x \in C$ with $A x \in Q$, if such $x$ exists. The split feasibility problem was first introduced in 1994 for modeling inverse problems that arise from phase retrievals and in medical image reconstruction. Many image reconstruction problems can be formulated as the split feasibility problem; see, for example, $[6,8]$ and the references therein.

Censor and Elfving [7] introduced and investigated the following CQ algorithm

$$
x_{0} \in R^{N}, x_{n+1}=\operatorname{Proj}_{C}^{R^{N}}\left(x_{n}-\delta A^{\top}\left(I-\operatorname{Proj}_{Q}^{R^{M}}\right) A x_{n}\right), \quad n \geqslant 0,
$$

where $\delta \in\left(0, \frac{2}{E}\right), E$ is the largest eigenvalue of the matrix $A^{\top} A$, and $I$ is the identity matrix.

Recently, Byrne [5] developed the split feasibility problem in the setting of infinite dimensional Hilbert spaces.

Let $C$ and $Q$ be nonempty, closed, and convex subsets in Hilbert spaces $\mathrm{H}_{1}$ and $\mathrm{H}_{2}$, respectively. Then the split feasibility problem in the framework of infinite dimensional spaces is formulated as finding a point $x \in C$ with the property:

$$
x \in C, A x \in Q,
$$

where $A: C \subset H_{1} \rightarrow H_{2}$ is a bounded linear operator. In view of the applications, the splitting feasibility problem has been studied by many authors in the framework of infinite dimensional Hilbert spaces; see $[2,9,17,20,22]$ and the references therein. 
We denote by $\operatorname{SFP}(A)$ the solution set of the split feasibility problem, that is,

$$
\operatorname{SFP}(A)=\left\{x \in H_{1}: x \in C, A x \in Q\right\}=A^{-1}(Q) \cap C .
$$

It is clear that $A^{-1}(Q)$ is a closed convex subset of $H_{1}$. Therefore, $\operatorname{SFP}(A)$ is also a closed convex subset of $\mathrm{H}_{1}$. It is known that the split feasibility problem is very general. It, which includes convex feasibility problem which is to find a common element in the intersection of a family of nonempty closed and convex subsets of a Hilbert space, has been extensively investigated; see $[9,12,15]$ and the references therein.

Let $\operatorname{Proj}_{\mathrm{C}}^{\mathrm{H}_{1}}$ and $\operatorname{Proj}_{\mathrm{Q}}^{\mathrm{H}_{2}}$ be metric projections onto sets $\mathrm{C}$ and $\mathrm{Q}$, respectively. It is well-known that if $\operatorname{SFP}(A) \neq \emptyset$, then solving split feasibility problem (1.1) is equivalent to solving a fixed point equation

$$
x=\operatorname{Proj}_{C}^{\mathrm{H}_{1}}\left(x-\delta A^{*}\left(\mathrm{I}-\operatorname{Proj}_{\mathrm{Q}}^{\mathrm{H}_{2}}\right) A x\right),
$$

where $\delta>0$ is a parameter and $A^{*}$ is the adjoint operator of $A$. If we define a mapping $U_{\delta}$ by

$$
\mathrm{U}_{\delta} x=x-\delta A^{*}\left(\mathrm{I}-\operatorname{Proj}_{\mathrm{Q}}^{\mathrm{H}_{2}}\right) A x,
$$

then one has $x=\operatorname{Proj}_{C}^{\mathrm{H}_{1}} \mathrm{U}_{\gamma} \chi$.

Assume that split feasibility problem (1.1) is consistent, i.e., the problem has a solution. It is easy to see that $\operatorname{Fix}\left(U_{\delta}\right)=A^{-1}(Q)$ and hence $\operatorname{SFP}(A)=C \cap \operatorname{Fix}\left(U_{\delta}\right)=\operatorname{Fix}\left(\operatorname{Proj}_{C}{ }_{C}{ }_{1} U_{\delta}\right)$, where $\operatorname{Fix}\left(U_{\delta}\right)$ and Fix $\left(\operatorname{Proj}_{C}^{\mathrm{H}_{1}} U_{\delta}\right)$ denote the fixed point set of $U_{\delta}$ and $\operatorname{Proj}_{C} \mathrm{H}_{1} U_{\delta}$, respectively, for sufficiently small $\delta>0$; see Wang, Zhou [20], Zhou [21] and Zhou, Wang [22] for the details.

Let $\mathrm{D}$ be a nonempty closed and convex subset of a real Hilbert space $\mathrm{H}$. Recall that a mapping $\mathrm{T}: \mathrm{D} \rightarrow \mathrm{D}$ is a contractive mapping if and only if there exists a constant $\alpha \in[0,1)$ such that

$$
\|\mathrm{T} x-\mathrm{T} y\| \leqslant \alpha\|x-y\|, \quad \forall x, y \in \mathrm{D} .
$$

$\mathrm{T}: \mathrm{D} \rightarrow \mathrm{D}$ is a nonexpansive mapping if and only if

$$
\|T x-T y\| \leqslant\|x-y\|, \quad \forall x, y \in D .
$$

From Browder [3], we know that the fixed point set of $T$ is not empty provided that $C$ is bounded, closed and convex. The theory of nonexpansive mappings has been recently applied to solve various convex optimization theories; see $[1,10,11,14,16,19]$ and the references therein. Recall that a mapping $\mathrm{S}: \mathrm{D} \rightarrow \mathrm{D}$ is said to be averaged if and only if it can be written as the average of the identity mapping and a nonexpansive mapping, i.e., $S:=(1-\alpha) I+\alpha S$ where $\alpha \in(0,1), S: D \rightarrow D$ is a nonexpansive mapping and $\mathrm{I}$ is the identity operator on D. We note that averaged mappings are nonexpansive. It is known that the composite of finitely many averaged mappings is still averaged. If the mappings $\left\{T_{i}\right\}_{i=1}^{N}$ are averaged and have a nonempty common fixed point set, then $\bigcap_{i=1}^{N} \operatorname{Fix}\left(T_{i}\right)=\operatorname{Fix}\left(T_{1}, T_{2}, \ldots, T_{N}\right)$.

It is well-known that if $\delta \in\left(0,2 /\|A\|^{2}\right)$, then $U_{\delta}$ is averaged and hence $\operatorname{Proj}_{C}^{\mathrm{H}_{1}} \mathrm{U}_{\delta}$ is also averaged, consequently, as a direct consequence of Reich's weak convergence theorem [18], the sequence $\left\{x_{n}\right\}$ is generated by the following procedure:

$$
x_{0} \in H_{1}, \quad x_{n+1}=\operatorname{Proj}_{C}^{H_{1}}\left[\left(I-\delta A^{*}\left(I-\operatorname{Proj}_{Q}^{\mathrm{H}_{2}}\right) A\right) x_{n}\right], \quad n \geqslant 0,
$$

where I denotes the identity mapping on $\mathrm{H}_{1}$ and $\mathrm{H}_{2}$, converges weakly to a solution of the feasibility problem; see Byrne [5] for the details. (1.2) is referred to as the Byrne's CQ algorithm in the existing literature.

Recall that $\mathrm{T}: \mathrm{D} \rightarrow \mathrm{D}$ is a strict pseudocontraction if and only if there exists a constant $\mathrm{k} \in[0,1)$ such that

$$
\|\mathrm{T} x-\mathrm{T} y\|^{2} \leqslant\|x-y\|+k\|(I-T) x-(I-T) y\|^{2}, \quad \forall x, y \in D .
$$


The class of strict pseudocontractions was introduced and investigated by Browder and Petryshyn [4] in 1967. It is clear that the class of strict pseudocontractions includes the class of nonexpansions as special cases.

Recall that a mapping $\mathrm{F}: \mathrm{D} \rightarrow \mathrm{H}$ is said to be monotone if and only if

$$
\langle F x-F y, x-y\rangle \geqslant 0, \quad \forall x, y \in D .
$$

$\mathrm{F}: \mathrm{D} \rightarrow \mathrm{H}$ is said to be $\mathrm{v}$-inverse strongly monotone if and only if

$$
\langle F x-F y, x-y\rangle \geqslant v\|F x-F y\|^{2}, \quad \forall x, y \in D .
$$

$\mathrm{F}: \mathrm{D} \rightarrow \mathrm{H}$ is said to be L-Lipschitzian if and only if $\|F x-F y\| \leqslant L\|x-y\|$ for all $x, y \in D$. We remark that if $F$ is $v$-inverse strongly monotone, then it is $\frac{1}{v}$-Lipschitzian and monotone. Let $T: D \rightarrow H$ be a nonexpansive mapping and define an operator $\mathrm{F}: \mathrm{D} \rightarrow \mathrm{H}$ by $\mathrm{Fx}=\mathrm{x}-\mathrm{T} x$. Then, $\mathrm{F}: \mathrm{D} \rightarrow \mathrm{H}$ is $\frac{1}{2}$-inverse strongly monotone.

Recently, many authors investigated the splitting feasibility problem in infinite dimensional Hilbert spaces via fixed point methods for the weak convergence of methods. In this paper, we consider a Halpern-like viscosity approximation method for the norm convergence of the method. The organization is as follows. In Section 2, some definitions and lemmas are provided. In Section 3, strong convergence theorems are established and some reduced results are also provided to support the main results.

\section{Preliminaries}

Let $\mathrm{D}$ be a nonempty closed and convex subset of a Hilbert space $\mathrm{H}$. Let $\mathrm{T}$ be a mapping. From now on, the fixed point set of $T$ will be denoted by $\operatorname{Fix}(T)$. For every point $x \in H$, there exists a unique nearest point in D denoted by $\operatorname{Proj}_{\mathrm{D}}^{\mathrm{H}} \mathrm{x}$ such that $\left\|x-\operatorname{Proj}_{\mathrm{D}}^{\mathrm{H}} \mathrm{x}\right\| \leqslant\|x-y\|$ for all $y \in \mathrm{D}$. Proj ${ }_{\mathrm{D}}^{\mathrm{H}}$ is called the metric projection of $\mathrm{H}$ onto D. It is well-known that $\operatorname{Proj}_{\mathrm{D}}^{\mathrm{H}}$ is nonexpansive mapping and satisfies $\left\langle x-y, \operatorname{Proj}_{\mathrm{D}}^{\mathrm{H}} x-\right.$ $\left.\operatorname{Proj}_{D}^{H} y\right\rangle \geqslant\left\|\operatorname{Proj}_{D}^{H} x-\operatorname{Proj}_{D}^{H} y\right\|^{2}$ for all $x, y \in H$. Moreover, $\operatorname{Proj}_{D}^{H} x$ is characterized by the fact $\operatorname{Proj}_{D}^{H} x \in D$ and $\left\langle x-\operatorname{Proj}_{D}^{\mathrm{H}} x, y-\operatorname{Proj}_{D}^{\mathrm{H}} x\right\rangle \leqslant 0$, and $\|x-y\|^{2} \geqslant\left\|x-\operatorname{Proj}_{D}^{\mathrm{H}} x\right\|^{2}+\left\|y-\operatorname{Proj}_{D}^{\mathrm{H}} x\right\|^{2}$ for all $x \in H, y \in \mathrm{D}$. In a real Hilbert space the following holds: $\|\lambda x+(1-\lambda) y\|^{2}=\lambda\|x\|^{2}+(1-\lambda)\|y\|^{2}-\lambda(1-\lambda)\|x-y\|^{2}$ for all $x, y \in H$ and $\lambda \in(0,1)$. It is well-known that every nonexpansive operator $T: H \rightarrow H$ satisfies, for all $x, y \in H \times H$, the inequality $\langle(x-T(x))-(y-T(y)), T(y)-T(x)\rangle \leqslant \frac{1}{2}\|(T(x)-x)-(T(y)-y)\|^{2}$, and therefore, we get, for all $(x, y) \in H \times \operatorname{Fix}(T),\langle x-T(x), y-T(y)\rangle \leqslant \frac{1}{2}\|T(x)-x\|^{2}$.

Lemma 2.1 ([13]). Let $\left\{a_{n}\right\}$ be a sequence of nonnegative real numbers such that

$$
a_{n+1} \leqslant\left(1-t_{n}\right) a_{n}+b_{n}+c_{n}, \quad \forall n \geqslant 0,
$$

where $\left\{c_{n}\right\}$ is a sequence of nonnegative real numbers, $\left\{t_{n}\right\} \subset(0,1)$, and $\left\{b_{n}\right\}$ is a sequence of real numbers. Assume that

(a) $\limsup _{n \rightarrow \infty} \frac{b_{n}}{t_{n}} \leqslant 0, \sum_{n=0}^{\infty} t_{n}=\infty$;

(b) $\sum_{n=0}^{\infty} c_{n}<\infty$.

Then $\lim _{n \rightarrow \infty} a_{n}=0$.

Lemma 2.2 ([3]). Let $\mathrm{H}$ be a Hilbert space and let $\mathrm{D}$ be a nonempty closed and convex subset of $\mathrm{H}$. Let $\mathrm{S}$ be a strict pseudocontraction on $\mathrm{D}$ with fixed points. If $x_{n} \rightarrow x^{*}$, where $\rightarrow$ denotes the weak convergence, and $\lim _{n \rightarrow \infty}\left\|x_{n}-T x_{n}\right\|=0$, then $x^{*}$ is a fixed point of $\mathrm{T}$, that is, $\mathrm{p}=\mathrm{Tp}$. In addition, $\operatorname{Fix}(\mathrm{T})$ is closed and convex.

The following two lemmas are known and not hard to derive.

Lemma 2.3. Let Proj $\mathrm{j}_{\mathrm{D}}^{\mathrm{H}}: \mathrm{H} \rightarrow \mathrm{D}$ be the metric projection from $\mathrm{H}$ on a nonempty, closed, and convex subset $\mathrm{D}$. Then the following conclusions hold true

(a) $\left\langle\left(\mathrm{I}-\operatorname{Proj}_{\mathrm{D}}^{\mathrm{H}}\right) x-\left(\mathrm{I}-\operatorname{Proj}_{\mathrm{D}}^{\mathrm{H}}\right) y, x-y\right\rangle \geqslant\left\|\left(\mathrm{I}-\operatorname{Proj}_{\mathrm{D}}^{\mathrm{H}}\right) \mathrm{x}-\left(\mathrm{I}-\operatorname{Proj}_{\mathrm{D}}^{\mathrm{H}}\right) y\right\|^{2}, \forall x, y \in \mathrm{H}$.

(b) $\|x-y\|^{2}-\left\|\left(I-\operatorname{Proj}_{D}^{\mathrm{H}}\right) x-\left(\mathrm{I}-\operatorname{Proj}_{\mathrm{D}}^{\mathrm{H}}\right) y\right\|^{2} \geqslant\left\|\operatorname{Proj}_{\mathrm{D}}^{\mathrm{H}} \mathrm{x}-\operatorname{Proj}_{\mathrm{D}}^{\mathrm{H}} \mathrm{y}\right\|^{2}, \forall x, y \in \mathrm{H}$

Lemma 2.4. Let $\mathrm{H}$ be a Hilbert space. Then the following inequality holds

$$
\|x+y\|^{2} \leqslant\|x\|^{2}+2\langle y, x+y\rangle, \quad \forall x, y \in H .
$$




\section{Main results}

Theorem 3.1. Let $\mathrm{H}_{1}$ and $\mathrm{H}_{2}$ be two real Hilbert spaces. Let $\mathrm{C}$ be a nonempty closed and convex subset of $\mathrm{H}_{1}$ and let $\mathrm{Q}$ be a nonempty closed and convex subset of $\mathrm{H}_{2}$. Let Proj $\mathrm{H}_{\mathrm{C}}^{\mathrm{H}_{1}}$ be the metric projection from $\mathrm{H}_{1}$ onto $\mathrm{C}$ and let Proj $_{\mathrm{Q}}^{\mathrm{H}_{2}}$ be the metric projection from $\mathrm{H}_{2}$ onto $\mathrm{Q}$. Let $\mathrm{f}: \mathrm{C} \rightarrow \mathrm{C}$ be a contractive mapping with constant $0 \leqslant \alpha<1$ and let $\mathrm{T}: \mathrm{C} \rightarrow \mathrm{C}$ be a strict pseudocontraction with constant $0 \leqslant \mathrm{~K}<1$. Let $\mathrm{A}: \mathrm{H}_{1} \rightarrow \mathrm{H}_{2}$ be a bounded linear operator such that split feasibility problem (1.1) is consistent. Assume that $\operatorname{Sol}(\mathrm{SFP}) \cap \operatorname{Fix}(\mathrm{S}) \neq \emptyset$. Let $\left\{\mathrm{x}_{\mathrm{n}}\right\}$ be a sequence generated in the following iterative algorithm

$$
\left\{\begin{array}{l}
x_{1} \in C \\
y_{n}=\gamma_{n} x_{n}+\left(1-\gamma_{n}\right) T x_{n}, \\
x_{n+1}=\alpha_{n} y_{n}+\left(1-\alpha_{n}\right) \operatorname{Proj}_{C}^{H_{1}}\left(\left(1-\beta_{n}\right)\left(x_{n}-\delta_{n} A^{*}\left(I-\operatorname{Proj}_{Q}^{H_{2}}\right) A x_{n}\right)+\beta_{n} f\left(x_{n}\right)\right), \quad n \geqslant 1,
\end{array}\right.
$$

where $\left\{\delta_{n}\right\}$ is a positive real sequence such that $\sum_{n=1}^{\infty}\left|\delta_{n}-\delta_{n+1}\right|<\infty, 0<\delta \leqslant \delta_{n} \leqslant \delta^{\prime}<\frac{2}{\|A\|^{2}}$, where $\delta$ and $\delta^{\prime}$ are two real numbers, $\left\{\alpha_{n}\right\},\left\{\beta_{n}\right\}$, and $\left\{\gamma_{n}\right\}$ are three real sequences in $(0,1)$ such that $0<\alpha \leqslant \alpha_{n} \leqslant \alpha^{\prime}<1$, $\sum_{n=1}^{\infty}\left|\alpha_{n}-\alpha_{n+1}\right|<\infty, \lim _{n \rightarrow \infty} \beta_{n}=0, \sum_{n=1}^{\infty} \beta_{n}=\infty, \sum_{n=1}^{\infty}\left|\beta_{n}-\beta_{n+1}\right|<\infty, 0<\gamma \leqslant \gamma_{n} \leqslant k<1$, where $\gamma$ is a constant in $(0,1), \sum_{n=1}^{\infty}\left|\gamma_{n}-\gamma_{n+1}\right|<\infty$. If $\operatorname{Fix}(T) \cap \operatorname{SFP}(A)$ is not empty, then $\left\{x_{n}\right\}$ converges strongly to a point $x^{*} \in \operatorname{Fix}(T) \cap \operatorname{SFP}(A)$ and $x^{*}$ is the unique solution to the variational inequality

$$
\left\langle f\left(x^{*}\right)-x^{*}, x-x^{*}\right\rangle \leqslant 0, \quad \forall x \in \operatorname{Fix}(T) \cap \operatorname{SFP}(A) .
$$

Proof. Note that the common solution set is not empty. Fixing $p \in \operatorname{Fix}(T) \cap \operatorname{SFP}(A)$, we find from Lemma 2.3 that

$$
\begin{aligned}
\left\|y_{n}-p\right\|^{2} & =\left\|\gamma_{n}\left(x_{n}-p\right)+\left(1-\gamma_{n}\right)\left(T x_{n}-p\right)\right\|^{2} \\
& =\gamma_{n}\left\|x_{n}-p\right\|^{2}-\gamma_{n}\left(1-\gamma_{n}\right)\left\|x_{n}-T x_{n}\right\|^{2}+\left(1-\gamma_{n}\right)\left\|T x_{n}-p\right\|^{2} \\
& \leqslant \gamma_{n}\left\|x_{n}-p\right\|^{2}-\gamma_{n}\left(1-\gamma_{n}\right)\left\|x_{n}-T x_{n}\right\|^{2}+\left(1-\gamma_{n}\right)\left(\left\|x_{n}-p\right\|^{2}+\kappa\left\|x_{n}-T x_{n}\right\|^{2}\right) \\
& \leqslant\left\|x_{n}-p\right\|^{2}-\left(1-\gamma_{n}\right)\left(\gamma_{n}-\kappa\right)\left\|x_{n}-T x_{n}\right\|^{2} .
\end{aligned}
$$

Since $\gamma_{n} \leqslant \kappa$, we find that

$$
\left\|y_{n}-p\right\| \leqslant\left\|x_{n}-p\right\|
$$

Define a mapping $\mathrm{W}: \mathrm{H}_{1} \rightarrow \mathrm{H}_{1}$ by

$$
W x=A^{*}\left(A x-\operatorname{Proj}_{\mathrm{Q}}^{\mathrm{H}_{2}} A x\right), \quad \forall x \in \mathrm{H}_{1} .
$$

Since I- $\operatorname{Proj}_{\mathrm{Q}}^{\mathrm{H}_{2}}$ is inverse-strongly monotone, we find that

$$
\begin{aligned}
\langle x-y, W x-W y\rangle & =\left\langle x-y, A^{*}\left(A x-\operatorname{Proj}_{Q}^{H_{2}} A x\right)-A^{*}\left(A y-\operatorname{Proj}_{Q}^{H_{2}} A y\right)\right\rangle \\
& =\left\langle A x-A y,\left(A x-\operatorname{Proj}_{Q}^{H_{2}} A x\right)-\left(A y-\operatorname{Proj}_{Q}^{H_{2}} A y\right)\right\rangle \\
& \geqslant\left\|\left(A x-\operatorname{Proj}_{Q}^{H_{2}} A x\right)-\left(A y-\operatorname{Proj}_{Q}^{H_{2}} A y\right)\right\|^{2} \\
& \geqslant \frac{1}{\|A\|^{2}}\|W x-W y\|^{2}, \quad \forall x, y \in H_{1} .
\end{aligned}
$$

This shows that $W$ is $\frac{1}{\|A\|^{2}}$-inverse-strongly monotone. It follows that

$$
\begin{aligned}
\left\|\left(I-\delta_{n} W\right) x-\left(I-\delta_{n} W\right) y\right\|^{2} & =\delta_{n}^{2}\|W x-W y\|^{2}-2 \delta_{n}\langle W x-W y, x-y\rangle+\|x-y\|^{2} \\
& \leqslant \delta_{n}^{2}\|W x-W y\|^{2}-\frac{2 \delta_{n}}{\|A\|^{2}}\|W x-W y\|^{2}+\|x-y\|^{2} \\
& =\delta_{n}\left(\delta_{n}-\frac{2}{\|A\|^{2}}\right)\|W x-W y\|^{2}+\|x-y\|^{2}
\end{aligned}
$$


Since $\delta_{n} \leqslant \frac{2}{\|A\|^{2}}$, we find that $\left(I-\mu_{n} W\right)$ is a nonexpansive mapping with

$$
\operatorname{Fix}\left(I-\mu_{n} W\right)=W^{-1}(0) .
$$

On the other hand, one has $W^{-1}(0)=A^{-1}(Q)$. Indeed, letting $x \in A^{-1}(Q)$, we find from the definition of $W$ that $x \in W^{-1}(0)$. This proves $A^{-1}(Q) \subset W^{-1}(0)$. Let $x \in W^{-1}(0)$, that is, $W x=0$. Since $\operatorname{Sol}(S F P) \cap$ $\operatorname{Fix}(T) \neq \emptyset$, we can take a point $y \in \operatorname{Fix}(T) \cap \operatorname{SFP}(A)$. This implies

$$
\operatorname{Proj}_{Q}^{\mathrm{H}_{2}} \mathrm{Ay}=\mathrm{Ay} \text { and } \mathrm{y}=\mathrm{Ty} \text {. }
$$

Hence, $W y=0$. Using (3.1), we have

$$
0=\langle x-y, W x-W y\rangle \geqslant\left\|\left(A x-\operatorname{Proj}_{Q}^{\mathrm{H}_{2}} A x\right)-\left(A y-\operatorname{Proj}_{Q}^{\mathrm{H}_{2}} A y\right)\right\|^{2}=\left\|\left(A x-\operatorname{Proj}_{Q}^{\mathrm{H}_{2}} A x\right)\right\|^{2},
$$

which implies that

$$
\left(\mathrm{I}-\operatorname{Proj}_{\mathrm{Q}}^{\mathrm{H}_{2}}\right) \mathrm{Ax}=0,
$$

that is, $x \in A^{-1}(Q)$. This shows that $W^{-1}(0) \subset A^{-1}(Q)$. Hence, one has $W^{-1}(0)=A^{-1}(Q)$. Since $C$, $Q$ are closed and convex, we see that $\operatorname{SFP}(A)$ is also closed and convex. Since $T$ is strictly pseudocontractive, we find that $\operatorname{Fix}(T)$ is closed and convex. Since $\operatorname{Proj}_{\operatorname{Fix}(T) \cap S F P(A)}^{\mathrm{H}_{1}} f$ is $\alpha$-contractive, we see that $\operatorname{Proj}_{\mathrm{Fix}(T) \cap S F P(A)}^{\mathrm{H}} \mathrm{f}$ has a unique fixed point. Next, we use $x^{*}$ to denote the unique fixed point, that is, $x^{*}=\operatorname{Proj}_{\operatorname{Fix}(T) \cap S F P}^{\mathrm{H}_{1}}(A) \mathrm{f}\left(x^{*}\right)$. Putting

$$
z_{n}=\operatorname{Proj}_{C}^{H_{1}}\left(\left(1-\beta_{n}\right)\left(x_{n}-\delta_{n} A^{*}\left(I-\operatorname{Proj}_{Q}^{H_{2}}\right) A x_{n}\right)+\beta_{n} f\left(x_{n}\right)\right),
$$

one has

$$
\begin{aligned}
\left\|z_{n}-x^{*}\right\| & \leqslant\left\|\left(\left(1-\beta_{n}\right)\left(x_{n}-\delta_{n} W x_{n}\right)+\beta_{n} f\left(x_{n}\right)\right)-x^{*}\right\| \\
& \leqslant\left\|\left(1-\beta_{n}\right)\left(\left(x_{n}-\delta_{n} W x_{n}\right)-\left(x^{*}-\delta_{n} W x^{*}\right)\right)+\beta_{n}\left(f\left(x_{n}\right)-x^{*}\right)\right\| \\
& \leqslant\left(1-\beta_{n}\right)\left\|\left(x_{n}-\delta_{n} W x_{n}\right)-\left(x^{*}-\delta_{n} W x^{*}\right)\right\|+\beta_{n}\left\|f\left(x_{n}\right)-f\left(x^{*}\right)\right\|+\beta_{n}\left\|f\left(x^{*}\right)-x^{*}\right\| \\
& \leqslant \beta_{n}\left\|f\left(x^{*}\right)-x^{*}\right\|+\left(1-\beta_{n}(1-\alpha)\right)\left\|x_{n}-x^{*}\right\| .
\end{aligned}
$$

It follows that

$$
\begin{aligned}
\left\|x_{n+1}-x^{*}\right\| & \leqslant \alpha_{n}\left\|y_{n}-x^{*}\right\|+\left(1-\alpha_{n}\right)\left\|z_{n}-x^{*}\right\| \\
& \leqslant\left(1-\beta_{n}\left(1-\alpha_{n}\right)(1-\alpha)\right)\left\|x_{n}-x^{*}\right\|+\beta_{n}\left(1-\alpha_{n}\right)(1-\alpha) \frac{\left\|f\left(x^{*}\right)-x^{*}\right\|}{1-\alpha} .
\end{aligned}
$$

By mathematical induction, we find that

$$
\left\|x_{n+1}-x^{*}\right\| \leqslant \max \left\{\frac{\left\|f\left(x^{*}\right)-x^{*}\right\|}{1-\alpha},\left\|x_{1}-x^{*}\right\|\right\} .
$$

This shows that $\left\{x_{n}\right\}$ is bounded, so are $\left\{y_{n}\right\}$ and $\left\{z_{n}\right\}$. Putting

$$
S_{n}=\gamma_{n} I+\left(1-\gamma_{n}\right) T
$$

we find that $\operatorname{Fix}\left(S_{n}\right)=\operatorname{Fix}(T)$ for each $n$ and

$$
\begin{aligned}
\left\|S_{n} x_{n}-S_{n} x_{n-1}\right\|^{2}= & \gamma_{n}\left\|x_{n}-x_{n-1}\right\|^{2}-\gamma_{n}\left(1-\gamma_{n}\right)\left\|\left(x_{n}-x_{n-1}\right)-\left(T x_{n}-T x_{n-1}\right)\right\|^{2} \\
& +\left(1-\gamma_{n}\right)\left\|T x_{n}-T x_{n-1}\right\|^{2} \\
\leqslant & \gamma_{n}\left(\gamma_{n}-1\right)\left\|\left(x_{n}-x_{n-1}\right)-\left(T x_{n}-T x_{n-1}\right)\right\|^{2}+\gamma_{n}\left\|x_{n}-x_{n-1}\right\|^{2} \\
& +\left(1-\gamma_{n}\right)\left(\left\|x_{n}-x_{n-1}\right\|^{2}+\kappa\left\|\left(x_{n}-x_{n-1}\right)-\left(T x_{n}-T x_{n-1}\right)\right\|^{2}\right) \\
\leqslant & \left\|x_{n}-x_{n-1}\right\|^{2}-\left(1-\gamma_{n}\right)\left(\gamma_{n}-\kappa\right)\left\|\left(x_{n}-x_{n-1}\right)-\left(T x_{n}-T x_{n-1}\right)\right\|^{2} \\
\leqslant & \left\|x_{n}-x_{n-1}\right\|^{2} .
\end{aligned}
$$


It follows that

$$
\begin{aligned}
\left\|y_{n}-y_{n-1}\right\| & \leqslant\left\|S_{n} x_{n}-S_{n} x_{n-1}\right\|+\left\|S_{n} x_{n-1}-S_{n-1} x_{n-1}\right\| \\
& \leqslant\left\|x_{n}-x_{n-1}\right\|+\left|\gamma_{n}-\gamma_{n-1}\right|\left\|x_{n-1}-T x_{n-1}\right\| .
\end{aligned}
$$

Since $\left(I-\delta_{n} W\right)$ is nonexpansive, we find that

$$
\begin{aligned}
\left\|\left(I-\delta_{n} W\right) x_{n}-\left(I-\delta_{n-1} W\right) x_{n-1}\right\| \leqslant & \left\|\left(I-\delta_{n} W\right) x_{n}-\left(I-\delta_{n} W\right) x_{n-1}\right\| \\
& +\left\|\left(I-\delta_{n} W\right) x_{n-1}-\left(I-\delta_{n-1} W\right) x_{n-1}\right\| \\
\leqslant & \left\|\left(I-\delta_{n} W\right) x_{n-1}-\left(I-\delta_{n-1} W\right) x_{n-1}\right\|+\left\|x_{n}-x_{n-1}\right\| \\
\leqslant & \left|\delta_{n}-\delta_{n-1}\right|\left\|W x_{n-1}\right\|+\left\|x_{n-1}-x_{n}\right\| .
\end{aligned}
$$

It follows that

$$
\begin{aligned}
\left\|z_{n}-z_{n-1}\right\| \leqslant & \|\left(\left(1-\beta_{n}\right)\left(x_{n}-\delta_{n} W x_{n}\right)+\beta_{n} f\left(x_{n}\right)\right) \\
& -\left(\left(1-\beta_{n-1}\right)\left(x_{n-1}-\delta_{n-1} W x_{n-1}\right)+\beta_{n-1} f\left(x_{n-1}\right)\right) \| \\
\leqslant & \left(1-\beta_{n}\right)\left\|\left(x_{n}-\delta_{n} W x_{n}\right)-\left(x_{n-1}-\delta_{n-1} W x_{n-1}\right)\right\|+\beta_{n} \| f\left(x_{n}\right)-f\left(x_{n-1} \|\right. \\
& +\left|\beta_{n}-\beta_{n-1}\right|\left\|W_{n-1} x_{n-1}-f\left(x_{n}\right)\right\| \\
\leqslant & \left(1-\beta_{n}\right)\left\|x_{n-1}-x_{n}\right\|+\left(1-\beta_{n}\right)\left|\delta_{n}-\delta_{n-1}\right|\left\|W x_{n-1}\right\|+\beta_{n} \| f\left(x_{n}\right)-f\left(x_{n-1} \|\right. \\
& +\left|\beta_{n}-\beta_{n-1}\right|\left\|W_{n-1} x_{n-1}-f\left(x_{n}\right)\right\| \\
\leqslant & \left(1-\beta_{n}(1-\alpha)\right)\left\|x_{n-1}-x_{n}\right\|+\left|\delta_{n}-\delta_{n-1}\right|\left\|W x_{n-1}\right\| \\
& +\left|\beta_{n}-\beta_{n-1}\right|\left\|W_{n-1} x_{n-1}-f\left(x_{n}\right)\right\| .
\end{aligned}
$$

In view of (3.2) and (3.3), we find that

$$
\begin{aligned}
\left\|x_{n+1}-x_{n}\right\| \leqslant & \alpha_{n}\left\|y_{n}-y_{n-1}\right\|+\left(1-\alpha_{n}\right)\left\|z_{n}-z_{n-1}\right\|+\left|\alpha_{n}-\alpha_{n-1}\right|\left\|y_{n-1}-z_{n-1}\right\| \\
\leqslant & \left(1-\left(1-\alpha_{n}\right) \beta_{n}(1-\alpha)\right)\left\|x_{n}-x_{n-1}|+| \gamma_{n}-\gamma_{n-1} \mid\right\| x_{n-1}-T x_{n-1} \| \\
& +\left|\delta_{n}-\delta_{n-1}\right|\left\|W x_{n-1}\right\|+\left|\beta_{n}-\beta_{n-1}\right|\left|W_{n-1} x_{n-1}-f\left(x_{n}\right)\left\|+\left|\alpha_{n}-\alpha_{n-1}\right|\right\| y_{n-1}-z_{n-1} \| .\right.
\end{aligned}
$$

Using Lemma 2.1 and the conditions imposed on $\left\{\alpha_{n}\right\},\left\{\beta_{n}\right\},\left\{\gamma_{n}\right\}$, and $\left\{\delta_{n}\right\}$, we find that

$$
\lim _{n \rightarrow \infty}\left\|x_{n+1}-x_{n}\right\|=0 \text {. }
$$

Since $W$ is $\frac{1}{\|A\|^{2}}$-inverse-strongly monotone, we find that

$$
\begin{aligned}
\left\|\left(I-\delta_{n} W\right) x_{n}-x^{*}\right\|^{2} & =\delta_{n}^{2}\left\|W x_{n}-W x^{*}\right\|^{2}+\left\|x_{n}-x^{*}\right\|^{2}-2 \delta_{n}\left\langle W x_{n}-W x^{*}, x_{n}-x^{*}\right\rangle \\
& \leqslant \delta_{n}^{2}\left\|W x_{n}-W x^{*}\right\|^{2}+\left\|x_{n}-x^{*}\right\|^{2}-\frac{2 \delta_{n}}{\|A\|^{2}}\left\|W x_{n}-W x^{*}\right\|^{2} \\
& =\left(\delta_{n}^{2}-\frac{2 \delta_{n}}{\|A\|^{2}}\right)\left\|W x_{n}-W x^{*}\right\|^{2}+\left\|x_{n}-x^{*}\right\|^{2} \\
& =\delta_{n}\left(\delta_{n}-\frac{2}{\|A\|^{2}}\right)\left\|W x_{n}\right\|^{2}+\left\|x_{n}-x^{*}\right\|^{2} .
\end{aligned}
$$

Since $\|\cdot\|^{2}$ is convex, we find from (3.5) that

$$
\begin{aligned}
\left\|x_{n+1}-x^{*}\right\|^{2} & \leqslant \alpha_{n}\left\|S_{n} x_{n}-x^{*}\right\|^{2}+\left(1-\alpha_{n}\right)\left\|z_{n}-x^{*}\right\|^{2} \\
& \leqslant \alpha_{n}\left\|x_{n}-x^{*}\right\|^{2}+\left(1-\alpha_{n}\right)\left\|\left(1-\beta_{n}\right)\left(x_{n}-\delta_{n} W x_{n}\right)+\beta_{n} f\left(x_{n}\right)-x^{*}\right\|^{2} \\
& \leqslant \alpha_{n}\left\|x_{n}-x^{*}\right\|^{2}+\left(1-\alpha_{n}\right)\left(1-\beta_{n}\right)\left\|\left(x_{n}-\delta_{n} W x_{n}\right)-x^{*}\right\|^{2}+\beta_{n}\left(1-\alpha_{n}\right)\left\|f\left(x_{n}\right)-x^{*}\right\|^{2} \\
& \leqslant\left(1-\alpha_{n}\right)\left(1-\beta_{n}\right) \delta_{n}\left(\delta_{n}-\frac{2}{\|A\|^{2}}\right)\left\|W x_{n}\right\|^{2}+\beta_{n}\left\|f\left(x_{n}\right)-x^{*}\right\|^{2}+\left(1-\beta_{n}\left(1-\alpha_{n}\right)\right)\left\|x_{n}-x^{*}\right\|^{2} .
\end{aligned}
$$


Hence, we have

$$
\begin{aligned}
\left(1-\alpha_{n}\right)\left(1-\beta_{n}\right) \delta_{n}\left(\frac{2}{\|A\|^{2}}-\delta_{n}\right)\left\|W x_{n}\right\|^{2} & \leqslant \beta_{n}\left\|f\left(x_{n}\right)-x^{*}\right\|^{2}+\left(1-\beta_{n}\left(1-\alpha_{n}\right)\right)\left\|x_{n}-x^{*}\right\|^{2}-\left\|x_{n+1}-x^{*}\right\|^{2} \\
& \leqslant \alpha_{n}\left\|f\left(x_{n}\right)-x^{*}\right\|^{2}+\left(\left\|x_{n}-x^{*}\right\|+\left\|x_{n+1}-x^{*}\right\|\right)\left\|x_{n+1}-x_{n}\right\| .
\end{aligned}
$$

Using the conditions imposed on $\left\{\alpha_{n}\right\},\left\{\beta_{n}\right\}$, and $\left\{\delta_{n}\right\}$, we find from (3.4) that

$$
\lim _{n \rightarrow \infty}\left\|W x_{n}\right\|=0 \text {. }
$$

Note that

$$
\begin{aligned}
x_{n}-S_{n} x_{n} & =x_{n}-x_{n+1}+\left(1-\alpha_{n}\right)\left(z_{n}-S_{n} x_{n}\right) \\
& =x_{n}-x_{n+1}+\left(1-\alpha_{n}\right)\left(z_{n}-x_{n}\right)+\left(1-\alpha_{n}\right)\left(x_{n}-S_{n} x_{n}\right) .
\end{aligned}
$$

It follows that

$$
\begin{aligned}
\left\|S_{n} x_{n}-x_{n}\right\| & \leqslant \frac{\left\|x_{n+1}-x_{n}\right\|}{\alpha_{n}}+\frac{1-\alpha_{n}}{\alpha_{n}}\left\|x_{n}-\operatorname{Proj}_{C}^{H_{1}}\left(\left(1-\beta_{n}\right)\left(x_{n}-\mu_{n} W x_{n}\right)+\beta_{n} f\left(x_{n}\right)\right)\right\| \\
& \leqslant \frac{\left\|x_{n+1}-x_{n}\right\|}{\alpha_{n}}+\frac{1-\alpha_{n}}{\alpha_{n}}\left\|x_{n}-\left(\left(1-\beta_{n}\right)\left(x_{n}-\mu_{n} W x_{n}\right)+\beta_{n} f\left(x_{n}\right)\right)\right\| \\
& \leqslant \frac{\left\|x_{n}+1-x_{n}\right\|}{\alpha_{n}}+\frac{1-\alpha_{n}}{\alpha_{n}}\left(\beta_{n}\left\|x_{n}-f\left(x_{n}\right)\right\|+\left(1-\beta_{n}\right)\left\|x_{n}-\left(x_{n}-\mu_{n} W x_{n}\right)\right\|\right) \\
& \leqslant \frac{\left\|x_{n+1}-x_{n}\right\|}{\alpha_{n}}+\frac{\left(1-\alpha_{n}\right) \beta_{n}}{\alpha_{n}}\left\|x_{n}-f\left(x_{n}\right)\right\|+\frac{\left(1-\alpha_{n}\right)\left(1-\beta_{n}\right) \mu_{n}}{\alpha_{n}}\left\|W x_{n}\right\| .
\end{aligned}
$$

In view of (3.4) and (3.6), we arrive at

$$
\lim _{n \rightarrow \infty}\left\|S_{n} x_{n}-x_{n}\right\|=0
$$

On the other hand, we have

$$
\left\|T x_{n}-x_{n}\right\| \leqslant\left\|x_{n}-S_{n} x_{n}\right\|+\left\|T x_{n}-S_{n} x_{n}\right\| \leqslant\left\|x_{n}-S_{n} x_{n}\right\|+\gamma_{n}\left\|T x_{n}-x_{n}\right\| .
$$

From the restriction imposed on $\left\{\gamma_{n}\right\}$ and (3.7), we find that

$$
\lim _{n \rightarrow \infty}\left\|T x_{n}-x_{n}\right\|=0 \text {. }
$$

Next, we show that

$$
\limsup _{n \rightarrow \infty}\left\langle z_{n}-x^{*}, f\left(x^{*}\right)-x^{*}\right\rangle \leqslant 0 .
$$

We take a subsequence $\left\{z_{n_{m}}\right\}$ of $\left\{z_{n}\right\}$ such that

$$
\lim _{m \rightarrow \infty}\left\langle f\left(x^{*}\right)-x^{*}, z_{n_{m}}-x^{*}\right\rangle=\limsup _{n \rightarrow \infty}\left\langle f\left(x^{*}\right)-x^{*}, z_{n}-x^{*}\right\rangle .
$$

Note that

$$
\begin{aligned}
\left\|x_{n}-z_{n}\right\| & \leqslant\left\|x_{n}-\left(1-\beta_{n}\right)\left(x_{n}-\delta_{n} A^{*}\left(I-\operatorname{Proj}_{Q}^{\mathrm{H}_{2}}\right) A x_{n}\right)-\beta_{n} f\left(x_{n}\right)\right\| \\
& \leqslant \beta_{n}\left\|f\left(x_{n}\right)-x_{n}\right\|+\left(1-\beta_{n}\right)\left\|\left(x_{n}-\delta_{n} W x_{n}\right)-x_{n}\right\| \\
& \leqslant \beta_{n}\left\|f\left(x_{n}\right)-x_{n}\right\|+\delta_{n}\left\|W x_{n}\right\| .
\end{aligned}
$$

In view of $\lim _{n \rightarrow \infty} \beta_{n}=0$, we find from (3.6) that

$$
\lim _{n \rightarrow \infty}\left\|x_{n}-z_{n}\right\|=0 \text {. }
$$

This proves that $\left\{z_{\mathfrak{n}}\right\}$ is bounded. This shows that $\left\{z_{\mathfrak{n}_{m}}\right\}$ is also bounded. We may assume that $\left\{z_{n_{m}}\right\}$ 
converges weakly to $z \in \mathrm{H}_{1}$. Since $\mathrm{C}$ is weakly closed, we see that $z \in \mathrm{C}$. Since

$$
\left\|T z_{n}-z_{n}\right\| \leqslant\left\|z_{n}-x_{n}\right\|+\left\|x_{n}-T x_{n}\right\|+\left\|T x_{n}-T z_{n}\right\|,
$$

we find from (3.9) and the Lipschitz continuity of $T$ that

$$
\lim _{n \rightarrow \infty}\left\|\mathrm{T} z_{n}-z_{n}\right\|=0 .
$$

From (3.6), we also have $\lim _{\mathfrak{n} \rightarrow \infty}\left\|W z_{\mathfrak{n}}\right\|=0$. Using Lemma 2.2, we find that $z$ is a fixed point of T. Since $W$ is inverse-strongly monotone, we have

$$
\frac{1}{\|A\|^{2}}\left\|W z_{n_{m}}-W z\right\|^{2} \leqslant\left\langle W z_{n_{m}}-W z, z_{n_{m}}-z\right\rangle
$$

Letting $\mathrm{m} \rightarrow \infty$ in (3.10), we find that $z \in \mathrm{W}^{-1}(0)$. This proves that

$$
z \in C \cap W^{-1}(0) \cap \operatorname{Fix}(T)=\operatorname{SFP}(A) \cap \operatorname{Fix}(T) .
$$

Using (3.8), one obtains that

$$
\limsup _{n \rightarrow \infty}\left\langle z_{n}-x^{*}, f\left(x^{*}\right)-x^{*}\right\rangle \leqslant 0 .
$$

Finally, we prove that $x_{n} \rightarrow x^{*}$ in norm as $n \rightarrow \infty$. Using Lemma 2.4, we find that

$$
\begin{aligned}
\left\|z_{n}-x^{*}\right\|^{2} & \leqslant\left\|\left(1-\beta_{n}\right)\left(x_{n}-\delta_{n} A^{*}\left(I-\operatorname{Proj}_{Q}^{H_{2}}\right) A x_{n}\right)+\beta_{n} f\left(x_{n}\right)-x^{*}\right\|^{2} \\
& \leqslant\left(1-\beta_{n}\right)^{2}\left\|\left(x_{n}-\delta_{n} A^{*}\left(I-\operatorname{Proj}_{Q}^{H_{2}}\right) A x_{n}\right)-x^{*}\right\|+2 \beta_{n}\left\langle f\left(x_{n}\right)-x^{*}, z_{n}-x^{*}\right\rangle \\
& \leqslant\left(1-\beta_{n}\right)^{2}\left\|x_{n}-x^{*}\right\|^{2}+2 \beta_{n} \alpha\left\|x_{n}-x^{*}\right\|\left\|z_{n}-x^{*}\right\|+2 \beta_{n}\left\langle f\left(x^{*}\right)-x^{*}, z_{n}-x^{*}\right\rangle \\
& \leqslant\left(1-2 \beta_{n}+\beta_{n}^{2}+\beta_{n} \alpha\right)\left\|x_{n}-x^{*}\right\|^{2}+\beta_{n} \alpha\left\|z_{n}-x^{*}\right\|^{2}+2 \beta_{n}\left\langle f\left(x^{*}\right)-x^{*}, z_{n}-x^{*}\right\rangle .
\end{aligned}
$$

It follows that

$$
\begin{aligned}
\left\|z_{n}-x^{*}\right\|^{2} \leqslant & \left(1-\frac{2 \beta_{n}(1-\alpha)}{1-\beta_{n} \alpha}\right)\left\|x_{n}-x^{*}\right\|^{2} \\
& +\frac{2 \beta_{n}(1-\alpha)}{1-\beta_{n} \alpha}\left(\frac{1}{1-\alpha}\left\langle f\left(x^{*}\right)-x^{*}, z_{n}-x^{*}\right\rangle+\frac{\beta_{n}}{2(1-\alpha)}\left\|x_{n}-x^{*}\right\|^{2}\right) .
\end{aligned}
$$

On the other hand, we have

$$
\begin{aligned}
\left\|x_{n+1}-x^{*}\right\|^{2} & \leqslant \alpha_{n}\left\|y_{n}-x^{*}\right\|^{2}+\left(1-\alpha_{n}\right)\left\|z_{n}-x^{*}\right\|^{2} \\
& \leqslant \alpha_{n}\left\|x_{n}-x^{*}\right\|^{2}+\left(1-\alpha_{n}\right)\left(1-\bar{\alpha}_{n}\right)\left\|x_{n}-x^{*}\right\|^{2}+\left(1-\alpha_{n}\right) \bar{\alpha}_{n} \lambda_{n} \\
& \leqslant\left(1-\bar{\alpha}_{n}\left(1-\alpha_{n}\right)\right)\left\|x_{n}-x^{*}\right\|^{2}+\bar{\alpha}_{n}\left(1-\alpha_{n}\right) \lambda_{n},
\end{aligned}
$$

where

$$
\lambda_{n}=\frac{\beta_{n}}{2(1-\alpha)}\left\|x_{n}-x^{*}\right\|^{2}+\frac{1}{1-\alpha}\left\langle f\left(x^{*}\right)-x^{*}, z_{n}-x^{*}\right\rangle .
$$

Since $\beta_{n} \rightarrow 0$ as $n \rightarrow \infty$ and $\left\{\left\|x_{n}-x^{*}\right\|\right\}$ is bounded, we find that $\lim _{\sup } \rightarrow \infty \lambda_{n} \leqslant 0$. Using Lemma 2.1, we find that $x_{n} \rightarrow x^{*}$ as $n \rightarrow \infty$. This completes the proof.

From Theorem 3.1, we immediately obtain the following result.

Corollary 3.2. Let $\mathrm{H}_{1}$ and $\mathrm{H}_{2}$ be two real Hilbert spaces. Let $\mathrm{C}$ be a nonempty closed and convex subset of $\mathrm{H}_{1}$ and let $\mathrm{Q}$ be a nonempty closed and convex subset of $\mathrm{H}_{2}$. Let Proj $\mathrm{H}_{\mathrm{C}}^{\mathrm{H}_{1}}$ be the metric projection from $\mathrm{H}_{1}$ onto $\mathrm{C}$ and let Proj $\mathrm{H}_{\mathrm{Q}}$ be the metric projection from $\mathrm{H}_{2}$ onto $\mathrm{Q}$. Let $\mathrm{f}: \mathrm{C} \rightarrow \mathrm{C}$ be a contractive mapping with constant $0 \leqslant \alpha<1$ and let $\mathrm{T}: \mathrm{C} \rightarrow \mathrm{C}$ be a nonexpansive mapping. Let $\mathrm{A}: \mathrm{H}_{1} \rightarrow \mathrm{H}_{2}$ be a bounded linear operator such that split feasibility problem (1.1) is consistent. Assume that $\operatorname{Sol}(\mathrm{SFP}) \cap \operatorname{Fix}(\mathrm{S}) \neq \emptyset$. Let $\left\{x_{n}\right\}$ be a sequence generated in the 
following iterative algorithm

$$
\left\{\begin{array}{l}
x_{1} \in C, \\
x_{n+1}=\alpha_{n} T x_{n}+\left(1-\alpha_{n}\right) \operatorname{Proj}_{C}^{H_{1}}\left(\left(1-\beta_{n}\right)\left(x_{n}-\delta_{n} A^{*}\left(I-\operatorname{Proj}_{Q}^{H_{2}}\right) A x_{n}\right)+\beta_{n} f\left(x_{n}\right)\right), n \geqslant 1,
\end{array}\right.
$$

where $\left\{\delta_{n}\right\}$ is a positive real sequence such that $\sum_{n=1}^{\infty}\left|\delta_{n}-\delta_{n+1}\right|<\infty, 0<\delta \leqslant \delta_{n} \leqslant \delta^{\prime}<\frac{2}{\|A\|^{2}}$, where $\delta$ and $\delta^{\prime}$ are two real numbers, $\left\{\alpha_{n}\right\}$ and $\left\{\beta_{n}\right\}$ are two real sequences in $(0,1)$ such that $0<\alpha \leqslant \alpha_{n} \leqslant \alpha^{\prime}<1$, $\sum_{n=1}^{\infty}\left|\alpha_{n}-\alpha_{n+1}\right|<\infty, \lim _{n \rightarrow \infty} \beta_{n}=0, \sum_{n=1}^{\infty} \beta_{n}=\infty, \sum_{n=1}^{\infty}\left|\beta_{n}-\beta_{n+1}\right|<\infty$. If $\operatorname{Fix}(T) \cap \operatorname{SFP}(A)$ is not empty, then $\left\{x_{n}\right\}$ converges strongly to a point $x^{*} \in \operatorname{Fix}(T) \cap \operatorname{SFP}(A)$ and $x^{*}$ is the unique solution to the variational inequality

$$
\left\langle f\left(x^{*}\right)-x^{*}, x-x^{*}\right\rangle \leqslant 0, \quad \forall x \in \operatorname{Fix}(T) \cap \operatorname{SFP}(A) .
$$

From Theorem 3.1, we also have the following results on splitting feasibility problem (1.1).

Corollary 3.3. Let $\mathrm{H}_{1}$ and $\mathrm{H}_{2}$ be two real Hilbert spaces. Let $\mathrm{C}$ be a nonempty closed and convex subset of $\mathrm{H}_{1}$ and let $\mathrm{Q}$ be a nonempty closed and convex subset of $\mathrm{H}_{2}$. Let $\operatorname{Proj}_{\mathrm{C}}^{\mathrm{H}_{1}}$ be the metric projection from $\mathrm{H}_{1}$ onto $\mathrm{C}$ and let Proj $_{\mathrm{Q}}^{\mathrm{H}_{2}}$ be the metric projection from $\mathrm{H}_{2}$ onto $\mathrm{Q}$. Let $\mathrm{f}: \mathrm{C} \rightarrow \mathrm{C}$ be a contractive mapping with constant $0 \leqslant \alpha<1$. Let $A: \mathrm{H}_{1} \rightarrow \mathrm{H}_{2}$ be a bounded linear operator such that split feasibility problem (1.1) is consistent. Let $\left\{x_{n}\right\}$ be a sequence generated in the following iterative algorithm

$$
\left\{\begin{array}{l}
x_{1} \in C, \\
x_{n+1}=\alpha_{n} x_{n}+\left(1-\alpha_{n}\right) \operatorname{Proj}_{C}^{H_{1}}\left(\left(1-\beta_{n}\right)\left(x_{n}-\delta_{n} A^{*}\left(I-\operatorname{Proj}_{Q}^{H_{2}}\right) A x_{n}\right)+\beta_{n} f\left(x_{n}\right)\right), \quad n \geqslant 1,
\end{array}\right.
$$

where $\left\{\delta_{n}\right\}$ is a positive real sequence such that $\sum_{n=1}^{\infty}\left|\delta_{n}-\delta_{n+1}\right|<\infty, 0<\delta \leqslant \delta_{n} \leqslant \delta^{\prime}<\frac{2}{\|A\|^{2}}$, where $\delta$ and $\delta^{\prime}$ are two real numbers, $\left\{\alpha_{n}\right\}$ and $\left\{\beta_{n}\right\}$ are two real sequences in $(0,1)$ such that $0<\alpha \leqslant \alpha_{n} \leqslant \alpha^{\prime}<1$, $\sum_{n=1}^{\infty}\left|\alpha_{n}-\alpha_{n+1}\right|<\infty, \lim _{n \rightarrow \infty} \beta_{n}=0, \sum_{n=1}^{\infty} \beta_{n}=\infty, \sum_{n=1}^{\infty}\left|\beta_{n}-\beta_{n+1}\right|<\infty$. If $\operatorname{SFP}(A)$ is not empty, then $\left\{x_{n}\right\}$ converges strongly to a point $x^{*} \in \operatorname{SFP}(A)$ and $x^{*}$ is the unique solution to the variational inequality

$$
\left\langle f\left(x^{*}\right)-x^{*}, x-x^{*}\right\rangle \leqslant 0, \quad \forall x \in \operatorname{SFP}(A) .
$$

Remark 3.4. The CQ algorithm heavily depends on metric projection $\operatorname{Proj}_{\mathrm{C}}$ and $\operatorname{Proj}_{\mathrm{Q}}$. In the framework of Hilbert spaces, the projections are nonexpansive. Indeed, they are firmly nonexpansive. However, they may lose the good properties in the framework of Banach spaces. It is of interest to extend the results presented in this article to a Banach space.

\section{Acknowledgment}

The authors are grateful to the editor and the referees for useful suggestions which improved the contents of this article.

\section{References}

[1] I. K. Argyros, S. George, Extending the applicability of a new Newton-like method for nonlinear equations, Commun. Optim. Theory, 2016 (2016), 9 pages. 1

[2] B. A. Bin Dehaish, X.-L. Qin, A. Latif, H.O. Bakodah, Weak and strong convergence of algorithms for the sum of two accretive operators with applications, J. Nonlinear Convex Anal., 16 (2015), 1321-1336. 1

[3] F. E. Browder, Existence and approximation of solutions of nonlinear variational inequalities, Proc. Nat. Acad. Sci. U.S.A., 56 (1966), 1080-1086. 1, 2.2

[4] F. E. Browder, W. V. Petryshyn, Construction of fixed points of nonlinear mappings in Hilbert space, J. Math. Anal. Appl., 20 (1967), 197-228. 1

[5] C. Byrne, A unified treatment of some iterative algorithms in signal processing and image reconstruction, Inverse Problems, 20 (2004), 103-120. 1, 1 
[6] Y. Censor, T. Bortfeld, B. Martin, A. Trofimov, A unified approach for inversion problems in intensity-modulated radiation therapy, Phys. Med. Biol., 51 (2006), 2353-2365. 1

[7] Y. Censor, T. Elfving, A multiprojection algorithm using Bregman projections in a product space, Numer. Algorithms, 8 (1994), 221-239. 1

[8] Y. Censor, T. Elfving, N. Kopf, T. Bortfeld, The multiple-sets split feasibility problem and its applications for inverse problems, Inverse Problems, 21 (2005), 2071-2084. 1

[9] S.-S. Chang, L. Wang, Y.-H. Zhao, On a class of split equality fixed point problems in Hilbert spaces, J. Nonlinear Var. Anal., 1 (2017), 201-212. 1

[10] S. Y. Cho, Strong convergence analysis of a hybrid algorithm for nonlinear operators in a Banach space, J. Appl. Anal. Comput. 8 (2018), 19-31. 1

[11] S. Y. Cho, B. A. Bin Dehaish, X.-L. Qin, Weak convergence of a splitting algorithm in Hilbert spaces, J. Appl. Anal. Comput., 7 (2017), 427-438. 1

[12] S. Y. Cho, X.-L. Qin, L. Wang, Strong convergence of a splitting algorithm for treating monotone operators, Fixed Point Theory Appl., 2014 (2014), 15 pages. 1

[13] L. S. Liu, Ishikawa and Mann iterative process with errors for nonlinear strongly accretive mappings in Banach spaces, J. Math. Anal. Appl., 194 (1995), 114-125. 2.1

[14] S.-T. Lv, Convergence analysis of a Halpern-type iterative algorithm for zero points of accretive operators, Commun. Optim. Theory, 2016 (2016), 9 pages. 1

[15] X.-L. Qin, S. Y. Cho, Convergence analysis of a monotone projection algorithm in reflexive Banach spaces, Acta Math. Sci. Ser. B Engl. Ed., 37 (2017), 488-502. 1

[16] X.-L. Qin, J.-C. Yao, Weak convergence of a Mann-like algorithm for nonexpansive and accretive operators, J. Inequal. Appl., 2016 (2016), 9 pages. 1

[17] X.-L. Qin, J.-C. Yao, Projection splitting algorithms for nonselfoperators, J. Nonlinear Convex Anal., 18 (2017), $925-935$. 1

[18] S. Reich, On fixed point theorems obtained from existence theorems for differential equations, J. Math. Anal. Appl., 54 (1976), 26-36. 1

[19] D. R. Sahu, J. C. Yao, A generalized hybrid steepest descent method and applications, J. Nonlinear Var. Anal., 1 (2017), 111-126. 1

[20] P.-Y. Wang, H.-Y. Zhou, A preconditioning method of the CQ algorithm for solving an extended split feasibility problem, J. Inequal. Appl., 2014 (2014), 11 pages. 1

[21] H.-Y. Zhou, Iterative methods of fixed points and zeros with applications, National Defence Industry Press, Beijing, (2016). 1

[22] H.-Y. Zhou, P.-Y. Wang, Adaptively relaxed algorithms for solving the split feasibility problem with a new step size, J. Inequal. Appl., 2014 (2014), 22 pages. 1 